\title{
Effect of Properties of Carbon Materials on Performance of VRLA Batteries
}

\author{
Ellappa Lakshmanan Nethaji, Kosaraju Srinivas, Kurivella Suryanarayana Murthy and Mandava Jagadish \\ $R \& A$ (Research \& Analysis), Technology Division, Amara Raja Batteries Limited, Karakambadi 517520, India
}

Received: September 09, 2015 / Accepted: October 08, 2015 / Published: November 30, 2015.

\begin{abstract}
In the present study, the relationship between properties of different carbon materials and their impact on performance of VRLA (valve regulated lead acid) battery was studied. The material properties undertaken for the study are: surface area, conductivity and water absorption of the carbon. The electrode morphology revealed the uniform distribution of active material when high surface area carbon was added to NAM (negative active material). The porosity of the plate also exhibited changes with respect to type of carbon materials added. The study further revealed that, the addition of high surface area carbon $\left(\sim 1,400 \mathrm{~m}^{2} / \mathrm{g}\right) \mathrm{improves}$ the charge acceptance of the battery with higher loading. Further improvement in charge acceptance was observed with addition of graphite to higher surface area carbon. Nevertheless, the float current of the battery got affected due to graphite loading and found there was no impact on shelf life of the battery in all the cases. The study demonstrates the need for customized "carbon formulation" to obtain the maximum performance out of the battery.
\end{abstract}

Key words: Valve regulated lead acid battery, carbon black, graphite, charge acceptance, float current, self discharge.

\section{Introduction}

The demand for mobile phone services in developing countries has radically increased the need for reliable telecommunication backup. Photovoltaic systems for solar electric power generation have also been fast emerging worldwide. These applications mainly rely on VRLA (valve regulated lead acid) battery technologies because of their versatility and lower cost compared to other energy storage technologies. Such applications however require VRLA batteries with robust performance such as higher charge acceptance, lower float charge current, improved cycle life, etc.

The NAM (negative active material) is critical and influences the charge acceptance and float current of lead acid battery to a large extent.

There have been several additives tried earlier to overcome the failure of NAM thereby improving the

Corresponding author: Kurivella Suryanarayana Murthy, general manager, research fields: lead acid battery and corrosion science. E-mail: ksnm@amararaja.co.in. charge acceptance of the battery [1]. Carbon is one of such additives extensively used to enhance the performance of NAM.

Moseley [2] established eight possible functions of carbon in enhancing the negative plate performance.

Sudhakar, et al. [3] proposed required qualities of NAM additives as (i) being inert to charge/discharge reactions, (ii) shall exhibit high stability in sulphuric acid medium and (iii) shall provide higher charge acceptance to the active material. Ellen, et al. [4] observed that, different carbon properties, such as degree of order, porosity, particle size, dry powder resistivity and impurities of carbon materials influence the cycle life of the battery significantly.

Jiayuan, et al. [5] have established that, prolonged HRPSoC (high rate partial state of charge) cycle life and enhanced charge acceptance can be achieved by adding 2 w.t. $\%$ of activated carbon to NAM. According to $\mathrm{Li}$, et al. [6], electrochemically active carbon can prolong cycle life of VRLA batteries under HRPSoC duty, but it may increase the evolution rate 
of hydrogen on the negative plates during the charging process. A few researchers suggested the addition of graphite enhances charge acceptance of batteries $[7,8]$ by elimination of surface build up of lead sulphate. Saravanan, et al. [9] proved that, replacing half of the spongy lead negative paste in negative plate with carbon plate has significantly improved the cycle life of lead-acid batteries.

Though literature discussed about the positive impacts of addition of carbon materials, their influence on battery performance metrics i.e., water loss, shelf life, float current, etc. is understood less. Further, the role of graphite along with carbon grades on lead acid battery performance is worth exploring. The main failure mode of lead acid battery in telecom application is due to water loss that can be attributed to higher float current. Similarly negative plate sulphation due to lower charge acceptance is observed to be the cause of failure mode in solar applications. The float current and charge acceptance of a battery are inversely related to each other. Thus, an optimal additive formulation is required to bring in workhorse lead acid battery technology to withstand in both telecom and solar applications. In our present work, carbon properties like surface area, resistivity and water absorption were correlated to the performance of the VRLA battery such as initial battery capacity, float current, shelf life and charge acceptance. Our main aim is to relate the properties of carbon materials required to improve charging capability of valve regulated lead acid batteries which is essential for telecom and SPV (solar photo voltaic) applications.

Three different carbon black grades possessing different surface areas were selected for the study. The advantage of addition of high conductive graphite along with these carbon black materials was established.

\section{Experimentation}

\subsection{Analytical Methods}

Different carbon blacks were characterized for surface area and active material resistivity. Water absorption of carbon sample was determined as to fix up the water content for lead paste preparation.

\subsubsection{Surface Area}

The surface area of different carbon materials were estimated by BET (Brauner-Emmett-Teller) method. The COULTER (model-SA3100) surface area analyzer was used for the said purpose.

\subsubsection{Water Absorption}

The water absorption of carbon powders was determined by following method. The carbon sample of $20 \mathrm{~g}$ was taken in a beaker and $50 \mathrm{~mL}$ of water was added to it, mixed well and cube weight of the carbon paste prepared was determined. The same procedure was continued with different water contents i.e., 60, $70,80,90$ and $100 \mathrm{~mL}$, respectively.

A plot was drawn between the amount of water added and cube weight of the carbon paste. The cube weight of the paste increased with increased water quantity initially, followed by a drop with subsequent water increase. The transition point of cube density i.e., high to low was correlated to the maximum amount of water that can absorb by the carbon material.

\subsubsection{NAM Electrical Resistivity}

The electrical resistivity of paste prepared with different carbon blacks was measured by four probe method [10]. Different carbon blacks along with other traditional additives were mixed with lead oxide. The electrodes were prepared by regular curing and formation methods. A pellet was collected from formed active materials and the electrical resistivity of the pellet was determined by four probe instrument (advanced model), Cromtech India.

\subsection{Battery Sample Preparation}

The standard industrial battery manufacturing facilities were used for preparation of sample cells. Optimum water content was added during the active material paste preparation, i.e., depending upon water absorption behavior of different carbon grades. 
Table 1 Carbon matrix adopted for study.

\begin{tabular}{|c|c|c|}
\hline S. No. & Grade & Weight percentage of carbon-graphite \\
\hline 1 & C1-0.2 & $0.2 \%-0 \%$ \\
\hline 2 & $\mathrm{C} 1-0.5$ & $0.5 \%-0 \%$ \\
\hline 3 & $\mathrm{C} 2-0.5$ & $0.5 \%-0 \%$ \\
\hline 4 & C $3-0.5$ & $0.5 \%-0 \%$ \\
\hline 5 & $\mathrm{C} 1-\mathrm{G}$ & $0.2 \%-0.2 \%$ \\
\hline 6 & $\mathrm{C} 2-\mathrm{G}$ & $0.2 \%-0.2 \%$ \\
\hline 7 & $\mathrm{C} 3-\mathrm{G}$ & $0.2 \%-0.2 \%$ \\
\hline
\end{tabular}

The prepared negative plates were assembled along with 4BS cured positive plates from the same batch of oxide. Experimental matrix for carbon study on $2 \mathrm{~V}-600 \mathrm{Ah}$ cells is as shown in Table 1.

\subsection{Active Material Morphology}

Batteries manufactured with different carbon grades were cut opened and SEM (scanning electron microscopy) was used to analyze the morphology of formed active material. The samples were sputter coated with a thin layer of gold, $\sim 10 \mathrm{~nm}$ in thickness, to reduce charging during analysis, thereby to have improved image quality. Analysis was carried out in a HITACHI-S3000N high vacuum model SEM. The plate composition i.e., lead, lead oxide and lead sulphate were also tested. The NAM plates were further examined for porosity.

\subsection{Electrical Testing}

The battery samples were subjected to performance tests namely initial capacity, float behavior, self discharge and charge acceptance. The tests were carried out with the help of Bitrode (model: LCN1-600-12) chargers. Parameters required for analysis like voltage, current, time and capacity were recorded automatically.

\section{Results \& Discussion}

\subsection{Material Properties}

\subsubsection{Surface Area}

Surface area of different carbon grades used in this study is determined by BET analysis and the results are shown Table 2.

The structure of particular carbon black depends on the level of linkage or branching of aggregate primary particles. Surface area of any carbon material is inversely proportional to its particles size. Ellen [11] describes that, the smaller the particles, the higher the tendency to create agglomerates in the electrodes. This obviously leads to less dispersed active material when small particle size carbons are used in paste preparation. A similar effect of uneven dispersion occurs when using large quantities of carbon materials.

However, uniformly distributed high surface area carbon is required to lower the charge voltage and to reduce pore radii of NAM as to overcome the localized growth of lead sulphate crystals during service life. Therefore, the choice of right particle size is all the more important, which would otherwise result in particle agglomeration and its uneven distribution in the paste as discussed above.

\subsubsection{Water Absorption}

Water absorption mainly depends on the physical properties of carbon such as particle size and its shape. The total water absorbed into the pore volume of the carbon particles and within the space between two carbon particles is determined.

Water absorption behavior can be observed from Fig. 1. It was observed that, the water absorption of the carbon increases with increase in surface area. Interestingly graphite has also shown higher water absorption despite its low surface area which can be attributed to lower apparent density and higher pore size.

Higher surface area enhances lead sulfate nucleation sites and also creates pores for transport of electrolyte into center of the electrode for improved active material utilization [12].

Table 2 Surface area of different carbon grades.

\begin{tabular}{lll}
\hline S. No. & Grade & BET $\left(\mathrm{m}^{2} / \mathrm{g}\right)$ \\
\hline 1 & $\mathrm{C} 1$ & 76 \\
2 & $\mathrm{C} 2$ & 220 \\
3 & $\mathrm{C} 3$ & 1,400 \\
4 & $\mathrm{G}$ & 28 \\
\hline
\end{tabular}




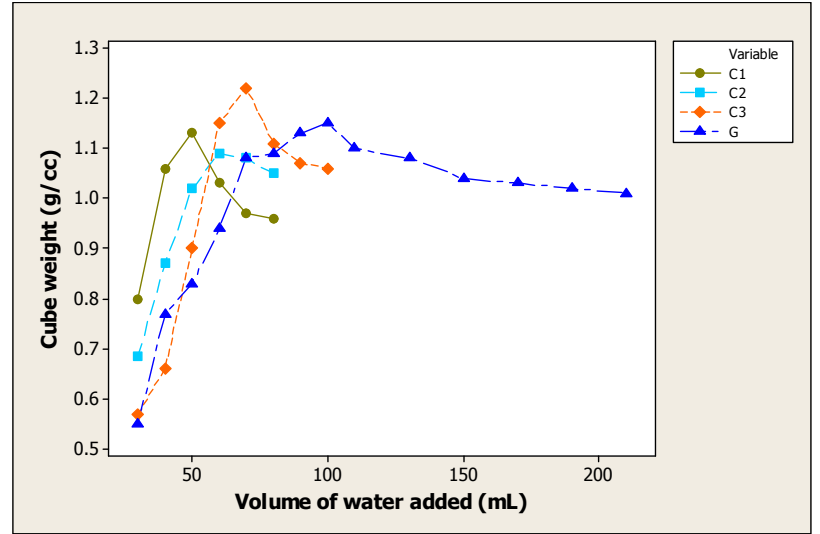

Fig. 1 Water absorption of different carbon grades.

\subsubsection{Electrical Resistivity}

When the battery is in partial state of charge, there are some regions occupied by lead sulphate which acts as an insulator. Carbon particles provide an electronically conducting pathway at this state [2]. The carbon having low electrical resistivity allows charging of the double layer. The potential barrier of electron transfer of $\mathrm{Pb}$ ions through carbon-solution interface is low and possible through higher conducting carbon. Some of the carbon types have a stable and low Ohmic contact with lead surface [13].

Therefore, the carbon addition to negative plates improve its conductivity thereby enhances charging efficiency. A pellet from formed plate was subjected to analysis by four probe method. The resistivity of different pellets is shown in Table 2. It was observed that, the resistivity of the active material increased with increase in surface area but it is not proportionate [5]. The resistivity decreased with percentage of carbon loading. The addition of graphite does also help to improve conductivity of the active material. Thus, it is helpful to add a highly conductive graphite material to get maximum conductivity of the active material.

\subsubsection{Active material morphology of Formed NAM}

The plate morphology of formed negative plates doped with different carbon chemistries is presented in Fig 2. It is clearly evident that, the addition of higher surface area carbon increases the porosity of the plates and active material is uniformly distributed (Fig. 2c). This helps in enhancement of lead sulfate nucleation sites and also allows transport of electrolyte into center of the electrode which improves active material utilization [5].

But addition of graphite (Fig. 2d) has reduced the overall porosity of the active material which cautions to use the graphite along with high surface area carbon only instead of graphite alone.

\subsection{Electrical Testing}

While the assessment of the material properties of the different carbon materials helped to understand the beneficial role of carbon, it is also essential to evaluate their performance at battery level. The important parameters assessed include: (a) initial capacity, (b) float current, (c) charge acceptance and (d) self-discharge.

Table 3 Electrical resistivity of formed NAM.

\begin{tabular}{lll}
\hline Grade & Notation & $\begin{array}{l}\text { Electrical resistivity } \\
(\mathrm{M} \Omega \cdot \mathrm{cm})\end{array}$ \\
\hline \multirow{3}{*}{1} & $\mathrm{C} 1-0.2$ & 450 \\
& $\mathrm{C} 1-0.5$ & 320 \\
& $\mathrm{C} 1-\mathrm{G}$ & 200 \\
$\mathrm{C} 2$ & $\mathrm{C} 2-0.2$ & 520 \\
& $\mathrm{C} 2-0.5$ & 400 \\
& $\mathrm{C} 2-\mathrm{G}$ & 260 \\
$\mathrm{C} 3$ & $\mathrm{C} 3-0.2$ & 1,220 \\
& $\mathrm{C} 3-0.5$ & 850 \\
& $\mathrm{C} 3-\mathrm{G}$ & 310 \\
\hline
\end{tabular}

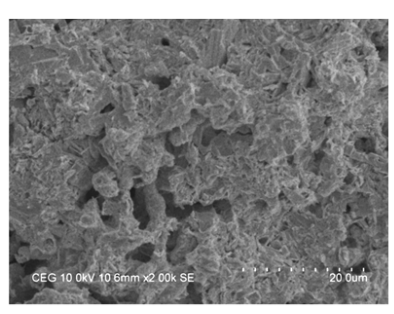

(a)

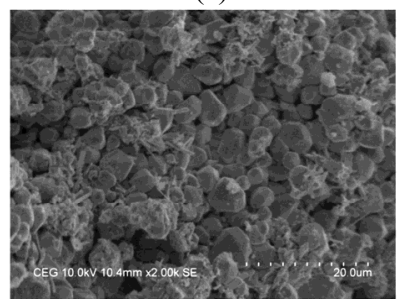

(c)

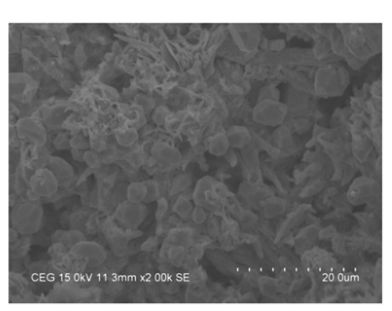

(b)

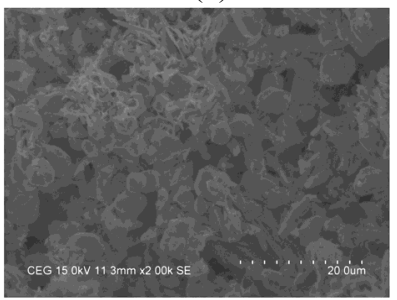

(d)
Fig. 2 SEM images of formed negative active material with different carbons: (a) C1-0.2; (b) C2-0.5; (c) C3-0.5; (d) C3-G. 


\subsubsection{Initial Capacity}

The batteries were initially boost charged at $2.3 \mathrm{~V}$ for $12 \mathrm{~h}$ and then discharged up to cut off voltage of $1.75 \mathrm{~V}$. The original test capacity is determined. As shown in Fig. 3, the delivered capacity of the batteries increased marginally with increase in surface area and also with carbon loading.

This is due to the improved active material with increase in surface area as discussed in Sections 3.1.2 and 3.1.4. The results are in correlation with studies done by Boden, et al. [8] where the carbon additives improved both initial capacity and cycle life of the battery. Thus there is no drawback in using carbon up to $0.5 \%$ of carbon as far as capacity is concerned.

\subsubsection{Float Current Behavior}

VRLA batteries in telecom applications mainly undergo float charge as battery is rarely utilized. Therefore, the float behavior is important to understand. The float charge current was measured by keeping the batteries at $2.25 \mathrm{~V}$ for 7 days and current accepted at the end of seventh day was noted.

Results show that, higher carbon loading and high surface area carbon marginally increase float current. Even low surface area carbon exhibited higher float current in the presence of graphite as shown in Fig. 4. This phenomenon is attributed to improved conductivity of active material due to addition of graphite as discussed in Section 3.1.3 which suppresses the voltage of the battery requiring higher float current to maintain the set voltage.

This cautions the usage of these combinations in float application as challenging one and requires further optimization and improvement.

\subsubsection{Self-Discharge}

One of the main drawbacks of lead acid batteries is its higher self-discharge. Normally, self-discharge is measured by two ways namely voltage drop per day and capacity loss for a given period of storage. In this study, the former method was chosen. The batteries were fully charged and then kept in open circuit condition for 60 days. The open circuit voltage of the

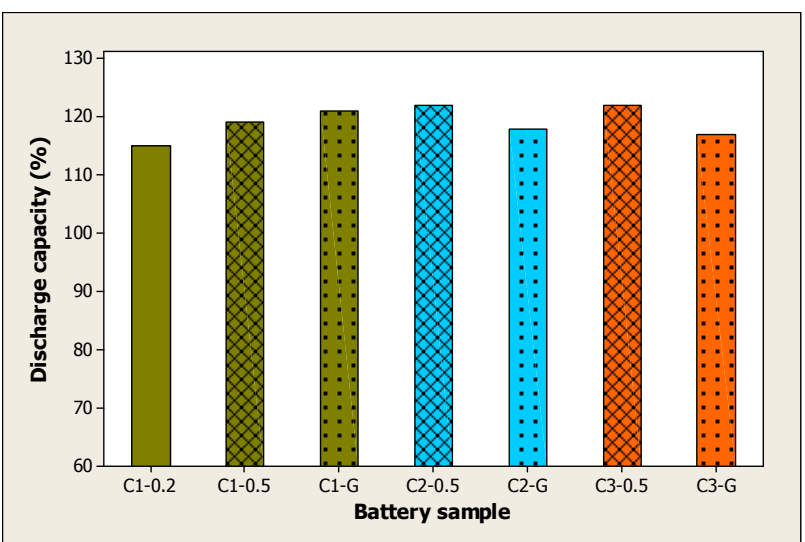

Fig. 3 Effect of different carbon materials on initial capacity.

test samples was measured every day. The average voltage drop over 60 day period is given in Fig. 5 .

The results show that, self-discharge of the batteries is not much affected due to addition of different carbon materials even with higher loading. This makes the usage of carbon material suitable for almost all application of lead acid batteries to take advantage of improved charge acceptance.

\subsubsection{Charge Acceptance}

Charge acceptance of a VRLA battery depends on type of carbon grade used in it. Dynamic charge acceptance is one way to indirectly measure charging behavior. Firstly, the battery was discharged up to $90 \%$ SOC (state of charge), soaked at $0{ }^{\circ} \mathrm{C}$ for $24 \mathrm{~h}$ and then charged at $2.4 \mathrm{~V}$ with $50 \%$ current limit. The current accepted at 10th $\mathrm{s}$ and 10th min of the charge step was noted down for comparison. It was observed that, the charge acceptance of the batteries improved slightly with respect to carbon loading and surface area. The addition of graphite along with high surface area carbon further improved charge acceptance of VRLA batteries as shown in Fig. 6.

The improvement in charge acceptance is due to: (a) the high surface area carbon is absorbed on to NAM surface uniformly thereby enhancing the reaction efficiency of the active material, (b) enhanced conductivity of the negative active material containing graphite as shown in Table 3.

This observation is in correlation with studies by 


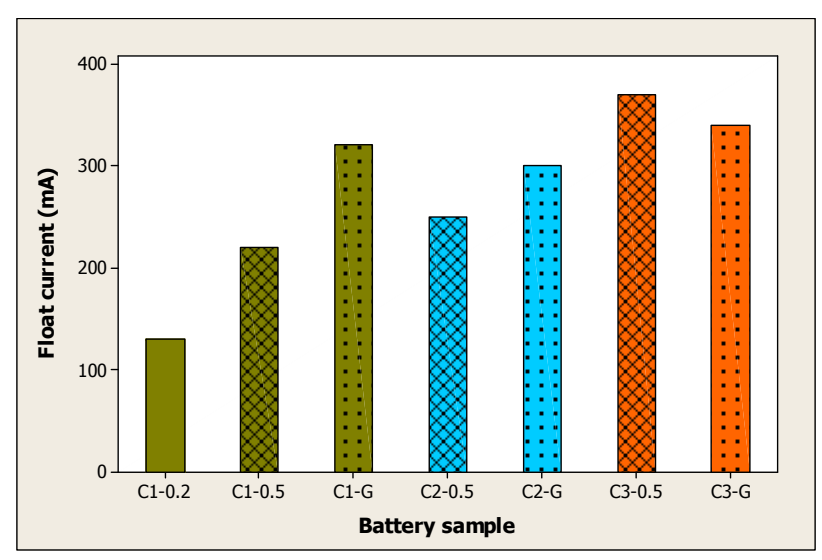

Fig. 4 Effect of different carbon materials on float current.

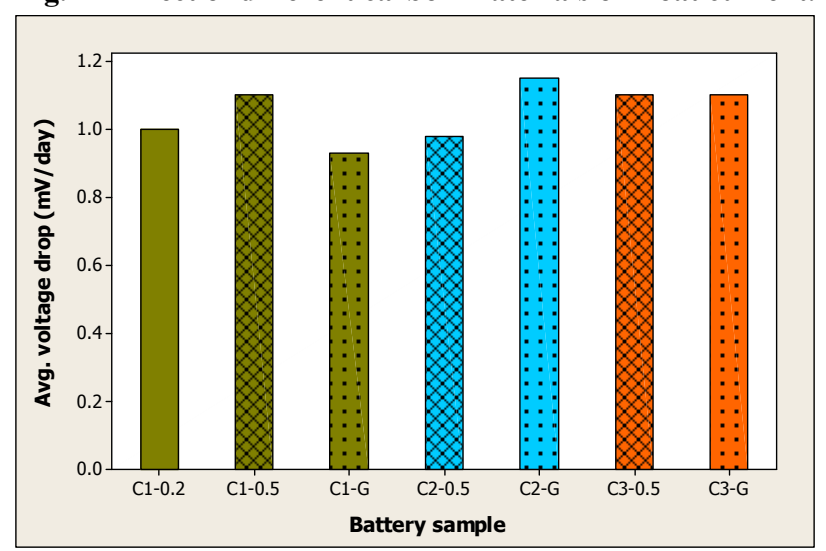

Fig. 5 Effect of different carbon materials on self discharge.

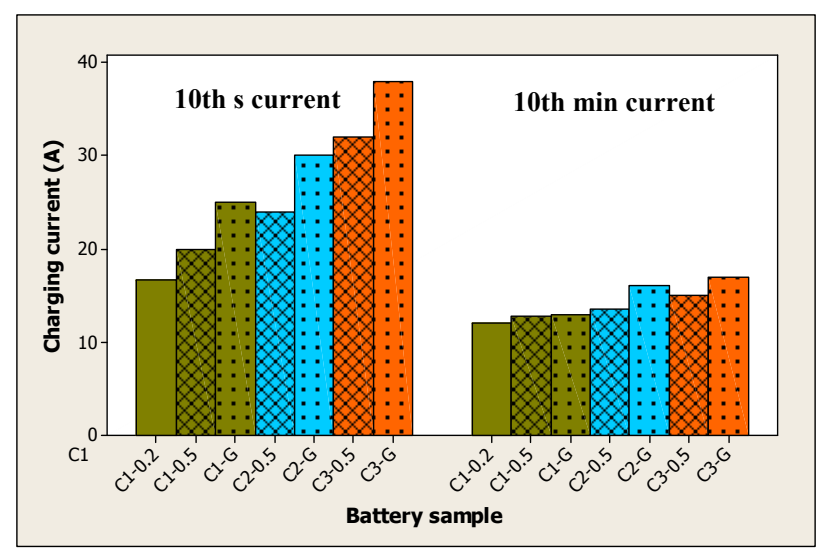

Fig. 6 Effect of different carbon materials on charge acceptance.

Fernandez, et al. [7] where they mainly concentrated on addition of graphite with different surface area carbon.

While short term electrical performance gives confidence in using carbon chemistry, endurance performance must be considered for complete understanding. The combination of high surface area and graphite may help in improvement of cycle life of the battery because of its uniform distribution overcoming failure of negative plate due to sulphation [14].

\section{Conclusions}

The followings are the main observations of the present study:

- The material properties of the carbon additive play important role in influencing performance of lead acid batteries;

- The addition of high surface area carbon increases the surface area of the active material and it does improve the charge acceptance of the battery;

- Addition of high conductive material such as graphite along with high surface area carbon further maximizes charge acceptance of the battery;

- The self discharge of the battery does not affected due to addition of carbon materials even with higher loading up to $0.5 \%$;

- The dosage of high surface area carbon can be limited up to $0.2 \%$ if the conductive material is added along with it;

- Addition of graphite leads to increase in float current. Thus one has to arrive at judicious ratio of graphite and high surface area carbon to get optimum performance.

\section{Acknowledgments}

The authors acknowledge with gratitude the resources and support provided by the Management of Amara Raja to carry out this study and the staff of Analytical Laboratory of Amara Raja Batteries Limited, Anna University, Chennai and Sri Venkateshwara University, Tirupati for helping in analyzing the samples.

\section{References}

[1] Rand, D. A. J., Moseley, P. T., Garche, J., and Parker, C. D. 2004. Valve Regulated Lead Acid Batteries. The Netherlands, USA, UK: Elsevier. 
[2] Moseley, P. T. 2009. "Consequences of Including Carbon in the Negative Plates of Valve-Regulated Lead-Acid Batteries Exposed to High-Rate Partial-State-of-Charge Operation." Journal of Power Sources 191 (1): 134-8.

[3] Sudhakar, J., Alpharetta, G. A., Fernandez, M., and Alcala de, H. M. 2012. Advanced graphite additive for enhanced cycle-life of lead-acid batteries. US Patent 2012/0,171,564 A1, filed January 4, 2011, and issued July 5, 2012.

[4] Ellen, E., Daniel, B., Jana, P., Alexander, B., Armin, F., Paolina, A., Jesus, V., Michael, W., and Eckart, R. 2013. "Carbon Blacks for Lead-Acid Batteries in Micro-Hybrid Applications Studied by Transmission Electron Microscopy and Raman Spectroscopy." Journal of Power Sources 222 (January): 554-60.

[5] Jiayuan, X., Ping, D., Hao, Z., Xianzhang, W., Jian, C., and Yusheng, Y. 2013. "Beneficial Effects of Activated Carbon Additives on the Performance of Negative Lead-Acid Battery Electrode for High-Rate Partial-State-of-Charge Operation." Journal of Power Sources 241 (November): 150-8.

[6] Li, Z., Baishuang, C., and Dianlong, W. 2013. "Effects of Electrochemically Active Carbon and Indium (III) Oxide in Negative Plates on Cycle Performance of Valve-Regulated Lead-Acid Batteries during High-Rate Partial-State-of-Charge Operation." Journal of Power Sources 231 (June): 34-8.

[7] Fernandez, M., Valenciano, J., Trinidad, F., and Munoz, N. 2010. "The Use of Activated Carbon and Graphite for the Development of Lead-Acid Batteries for Hybrid Vehicle Applications.” Journal of Power Sources 195 (14): 4458-69.

[8] Boden, D. P., Loosemore, D. V., Spence, M. A., and
Wojcinski, T. D. 2010. "Optimization Studies of Carbon Additives to Negative Active Material for the Purpose of Extending the Life of VRLA Batteries in High-Rate Partial-State-of-Charge Operation." Journal of Power Sources 195 (14): 4470-93.

[9] Saravanan, M., Ganesan, M., and Ambalavanan, S. 2012. "A Modified Lead-Acid Negative Electrode for High-Rate Partial-State-of-Charge Applications." Journal of the Electrochemical Society 159 (4): A452-A458.

[10] Panta, G. P., and Subedi, D. P. 2012. "Electrical Characterization of Aluminum (Al) Thin Films Measured by Using Four- Point Probe Method." Kathmandu University Journal of Science, Engineering and Technology 8 (December): 31-6.

[11] Ellen, E., Daniel, B., Alexander, B., Michael, W., Paolina, A., and Jesús, V. 2013. "Carbon Blacks for the Extension of the Cycle Life in Flooded Lead Acid Batteries for Micro-Hybrid Applications." Journal of Power Sources 239 (October): 483-9.

[12] Lead, A. T., Aaron, M. F., Henry, R. C., Alan, T. C., Katharine, G., and Avery, S. 2013. Carbon-lead blends for use in hybrid energy storage devices. US Patent 2013/0,004,841 A1, filed Junuary 1, 2012, and issued Junuary 3, 2013.

[13] Pavlov, D., Nikolov, P., and Rogachev, T. 2010. "Influence of Expander Components on the Processes at the Negative Plates of Lead-Acid Cells on High-Rate Partial-State-of-Charge Cycling. Part II. Effect of Carbon Additives on the Processes of Charge and Discharge of Negative Plates." Journal of Power Sources 195 (14): 4444-57.

[14] Detchko, P. 2011. Lead Acid Batteries: Science \& Technology. The Netherlands, UK: Elsevier. 